\author{
Pirzada S. ${ }^{1}$, Ganie H. A. ${ }^{1}$, Alghamdi A. M. ${ }^{2}$
}

\title{
ON THE SUM OF SIGNLESS LAPLACIAN SPECTRA OF GRAPHS
}

For a simple graph $G(V, E)$ with $n$ vertices, $m$ edges, vertex set $V(G)=\left\{v_{1}, v_{2}, \ldots, v_{n}\right\}$ and edge set $E(G)=\left\{e_{1}, e_{2}, \ldots, e_{m}\right\}$, the adjacency matrix $A=\left(a_{i j}\right)$ of $G$ is a $(0,1)$-square matrix of order $n$ whose $(i, j)$-entry is equal to 1 if $v_{i}$ is adjacent to $v_{j}$ and equal to 0 , otherwise. Let $D(G)=\operatorname{diag}\left(d_{1}, d_{2}, \ldots, d_{n}\right)$ be the diagonal matrix associated to $G$, where $d_{i}=\operatorname{deg}\left(v_{i}\right)$, for all $i \in$ $\{1,2, \ldots, n\}$. The matrices $L(G)=D(G)-A(G)$ and $Q(G)=D(G)+A(G)$ are respectively called the Laplacian and the signless Laplacian matrices and their spectra (eigenvalues) are respectively called the Laplacian spectrum ( $L$-spectrum) and the signless Laplacian spectrum ( $Q$-spectrum) of the graph $G$. If $0=\mu_{n} \leq \mu_{n-1} \leq \cdots \leq \mu_{1}$ are the Laplacian eigenvalues of $G$, Brouwer conjectured that the sum of $k$ largest Laplacian eigenvalues $S_{k}(G)$ satisfies $S_{k}(G)=\sum_{i=1}^{k} \mu_{i} \leq m+\left(\begin{array}{c}k+1 \\ 2\end{array}\right)$ and this conjecture is still open. If $q_{1}, q_{2}, \ldots, q_{n}$ are the signless Laplacian eigenvalues of $G$, for $1 \leq k \leq n$, let $S_{k}^{+}(G)=\sum_{i=1}^{k} q_{i}$ be the sum of $k$ largest signless Laplacian eigenvalues of $G$. Analogous to Brouwer's conjecture, Ashraf et al. conjectured that $S_{k}^{+}(G) \leq m+\left(\begin{array}{c}k+1 \\ 2\end{array}\right)$, for all $1 \leq k \leq n$. This conjecture has been verified in affirmative for some classes of graphs. We obtain the upper bounds for $S_{k}^{+}(G)$ in terms of the clique number $\omega$, the vertex covering number $\tau$ and the diameter of the graph $G$. Finally, we show that the conjecture holds for large families of graphs.

Key words and phrases: signless Laplacian spectra, Brouwer's conjecture, clique number, vertex covering number, diameter.

\footnotetext{
${ }^{1}$ University of Kashmir, 190006, Srinagar, India

${ }^{2}$ Umm Alqura University, P.O.Box 715, Makkah, Saudi Arabia

E-mail: pirzadasd@kashmiruniversity.ac.in (Pirzada S.), hilahmad1119kt@gmail.com (Ganie H. A.), amghamdi @uqu.edu.sa (Alghamdi A. M.)
}

\section{INTRODUCTION}

Let $G(V, E)$ be a simple graph with $n$ vertices, $m$ edges, having vertex set $V(G)=\left\{v_{1}, v_{2}, \ldots, v_{n}\right\}$ and edge set $E(G)=\left\{e_{1}, e_{2}, \ldots, e_{m}\right\}$. The adjacency matrix $A=\left(a_{i j}\right)$ of $G$ is a $(0,1)$-square matrix of order $n$ whose $(i, j)$-entry is equal to 1 if $v_{i}$ is adjacent to $v_{j}$ and equal to 0 , otherwise. Let $D(G)=\operatorname{diag}\left(d_{1}, d_{2}, \ldots, d_{n}\right)$ be the diagonal matrix associated to $G$, where $d_{i}=\operatorname{deg}\left(v_{i}\right)$, for all $i \in\{1,2, \ldots, n\}$. The matrices $L(G)=D(G)-A(G)$ and $Q(G)=D(G)+A(G)$ are respectively called the Laplacian and the signless Laplacian matrices and their spectra (eigenvalues) are respectively called the Laplacian spectrum ( $L$ spectrum) and the signless Laplacian spectrum ( $Q$-spectrum) of the graph $G$. These matrices are real symmetric and positive semi-definite. We let $0=\mu_{n} \leq \mu_{n-1} \leq \cdots \leq \mu_{1}$ and $0 \leq q_{n} \leq q_{n-1} \leq \cdots \leq q_{1}$ to be the $L$-spectrum and $Q$-spectrum of $G$, respectively. It is well

$\mathrm{Y} \Delta \mathrm{K} 519.17$

2010 Mathematics Subject Classification: 05C30,05C50.

The research of S. Pirzada (first author) is supported by SERB-DST, New Delhi under the research project number MTR/2017/000084. 
known that the multiplicity of the Laplacian eigenvalue $\mu_{n}=0$ is equal to the number of connected components of $G$ and also $\mu_{n-1}>0$ if and only if $G$ is connected. Moreover $\mu_{i}=q_{i}$, for all $i \in\{1,2, \ldots, n\}$, if and only if $G$ is bipartite [4].

For $k \in\{1,2, \ldots, n\}$, let $S_{k}(G)=\sum_{i=1}^{k} \mu_{i}$ be the sum of $k$ largest Laplacian eigenvalues of $G$. Also, let $d_{i}^{*}(G)=\left|\left\{v \in V(G): d_{v} \geq i\right\}\right|$, for $i \in\{1,2, \ldots, n\}$. In 1994, Grone and Merris [12] observed that for any graph $G$ and for any $k \in\{1,2, \ldots, n\}$,

$$
S_{k}(G) \leq \sum_{i=1}^{k} d_{i}^{*}(G) \text {. }
$$

This observation was proved by Hua Bai [2] and is nowadays called as Grone-Merris theorem. As an analogue to Grone-Merris theorem, Andries Brouwer [3] conjectured that for a graph $G$ with $n$ vertices and $m$ edges and for any $k \in\{1,2, \ldots, n\}$,

$$
S_{k}(G)=\sum_{i=1}^{k} \mu_{i} \leq m+\left(\begin{array}{c}
k+1 \\
2
\end{array}\right) .
$$

This conjecture is still open and is presently an active component of research. For the progress on this conjecture and related results, we refer to [8-11,14] and the references therein.

For $k \in\{1,2, \ldots, n\}$, let $S_{k}^{+}(G)=\sum_{i=1}^{k} q_{i}$ be the sum of $k$ largest signless Laplacian eigenvalues of a graph $G$. Motivated by the definition of $S_{k}(G)$ and Brouwer's conjecture, Ashraf et al. [1] proposed the following conjecture about $S_{k}^{+}(G)$.

Conjecture 1. If $G$ is a graph with $n$ vertices and $m$ edges, then

$$
S_{k}^{+}(G)=\sum_{i=1}^{k} q_{i} \leq m+\left(\begin{array}{c}
k+1 \\
2
\end{array}\right)
$$

for all $k \in\{1,2, \ldots, n\}$.

Using computations on a computer Ashraf et al. [1] verified the truth of this conjecture for all graphs with at most 10 vertices. For $k=1$, the conjecture follows from the well-known inequality $q_{1}(G) \leq \frac{2 m}{n-1}+n+2$ and $m \geq n-1$. Also, the cases $k=n$ and $k=n-1$ are straightforward. The conjecture is true for trees. This follows from the fact that Brouwer's conjecture holds for trees and that both Laplacian and signless Laplacian eigenvalues are the same for trees. Ashraf et al. [1] showed that the conjecture is true for all graphs when $k=2$ and is also true for regular graphs. Yang et al. [16] obtained various upper bounds for $S_{k}^{+}(G)$ and proved that the conjecture is also true for unicyclic graphs, bicyclic graphs and tricyclic graphs (except for $k=3$ ). For the progress on this conjecture and related results, we refer to $[1,7,16]$ and the references therein.

A clique of a graph $G$ is the maximum complete subgraph of the graph $G$. The order of the maximum clique is called the clique number of the graph $G$ and is denoted by $\omega$. A subset $S$ of the vertex set $V(G)$ is said to be a covering set of $G$ if every edge of $G$ is incident to at least one vertex in $S$. A covering set with minimum cardinality among all covering sets is called minimum covering set of $G$ and its cardinality, denoted by $\tau$, is called vertex covering number of $G$. 
The distance between any two vertices $u$ and $v$ is defined as the length of shortest path between them and the diameter of a graph $G$ is the maximum distance among all pair of vertices of $G$. If $H$ is a subgraph of the graph $G$, we denote the graph obtained by removing the edges in $H$ from $G$ by $G \backslash H$ (that is, only the edges of $H$ are removed from $G$ ).

Further, as usual $P_{n}, K_{n}$ and $K_{s, t}$, respectively, denote the path on $n$ vertices, the complete graph on $n$ vertices and the complete bipartite graph on $s+t$ vertices. For other undefined notations and terminology from spectral graph theory, the readers are referred to $[4,13]$.

The paper is organized as follows. In Section 2, we obtain some upper bounds for $S_{k}^{+}(G)$ in terms of the clique number $\omega$, the vertex covering number $\tau$ and the diameter of the graph G. As applications to the results obtained in Section 2, we prove that Conjecture 1 is true for some new classes of graphs in Section 3.

\section{UPPER BOUNDS FOR $S_{k}^{+}(G)$}

In this section, we obtain the upper bounds for $S_{k}^{+}(G)$, in terms of the clique number $\omega$, the vertex covering number $\tau$ and the diameter of the graph $G$.

Yang et al. [16] obtained the following upper bound for $S_{k}^{+}(G)$, in terms of the clique number $\omega$ and the number of edges $m$ :

$$
S_{k}^{+}(G) \leq k(\omega-2)+2 m-\omega(\omega-2)
$$

Das et al. [5] obtained an upper for $S_{k}(G)$ of a graph with $n$ vertices, in terms of the vertex covering number $\tau$ and the number of edges $m$. Using similar analysis, the following upper bound can be obtained for $S_{k}^{+}(G)$, in terms of the vertex covering number $\tau$ and the number of edges $m$ :

$$
S_{k}^{+}(G) \leq m+k \tau,
$$

with equality if and only if $G \cong K_{1, n-1}$.

The following observation is due to Fulton [6].

Lemma 1. Let $A$ and $B$ be two real symmetric matrices of order $n$. Then for any $1 \leq k \leq n$,

$$
\sum_{i=1}^{k} \lambda_{i}(A+B) \leq \sum_{i=1}^{k} \lambda_{i}(A)+\sum_{i=1}^{k} \lambda_{i}(B),
$$

where $\lambda_{i}(X)$ is the $i^{\text {th }}$ eigenvalue of the matrix $X$.

Let $\Gamma_{1}$ be the family of all connected graphs except for the graphs $G$, where the vertices in the vertex covering set $S=\left\{v_{1}, v_{2}, \ldots, v_{\omega-1}\right\}$ of the subgraph $K_{\omega}$ have the property that there are pendent vertices incident to some $v_{i} \in S$ or any two vertices of $S$ forms a triangle with a vertex $v \in V(G) \backslash C$, where $C$ is the vertex covering set of $G$.

The following theorem gives an upper bound for $S_{k}^{+}(G)$ in terms of the clique number $\omega$, the vertex covering number $\tau$ and the number of edges $m$ of the graph $G$. The number of vertices in a graph $G$ is denoted by $n(G)$ and the number of vertices adjacent to a vertex $v$ is denoted by $N(v)$. 
Theorem 2. Let $G \in \Gamma_{1}$ be a connected graph of order $n \geq 2$ with $m$ edges having clique number $\omega$ and vertex covering number $\tau$. Then, for $1 \leq k \leq n$,

$$
S_{k}^{+}(G) \leq k(\tau-1)+m-\frac{\omega(\omega-3)}{2},
$$

with equality if and only if $G \cong K_{n}$.

Proof. If $G \in \Gamma_{1}$ is a connected graph with clique number $\omega$, vertex cover number $\tau$ and minimum vertex covering set $C=\left\{v_{1}, v_{2}, \ldots, v_{\tau}\right\}$, then $K_{\omega}$ is a subgraph of $G$. Further, the vertex covering number of a complete graph on $\omega$ vertices is $\omega-1$. Without loss of generality, let $v_{1}, v_{2}, \ldots, v_{\omega-1}$ be the vertices in $C$, which belong to $V\left(K_{\omega}\right)$. The signless Laplacian spectrum of $K_{\omega}$ is $\left\{2 \omega-2, \omega-2^{[\omega-1]}\right\}$. After removing the edges of $K_{\omega}$ from $G$, the signless Laplacian matrix of $G$ isdecomposed as

$$
Q(G)=Q\left(K_{\omega} \cup(n-\omega) K_{1}\right)+Q\left(G \backslash K_{\omega}\right),
$$

where $G \backslash K_{\omega}$ is the graph obtained from $G$ by removing the edges of $K_{\omega}$. Using Lemma 1 and the fact $S_{k}^{+}\left(K_{\omega} \cup(n-\omega) K_{1}\right)=S_{k}^{+}\left(K_{\omega}\right)$, we have

$$
\begin{aligned}
S_{k}^{+}(G) & =\sum_{i=1}^{k} q_{i}(G) \leq \sum_{i=1}^{k} q_{i}\left(K_{\omega}\right)+\sum_{i=1}^{k} q_{i}\left(G \backslash K_{\omega}\right) \\
& =S_{k}^{+}\left(K_{\omega}\right)+S_{k}^{+}\left(G \backslash K_{\omega}\right)=\omega(k+1)-2 k+S_{k}^{+}\left(G \backslash K_{\omega}\right) .
\end{aligned}
$$

To complete the proof, we need to estimate $S_{k}^{+}\left(G \backslash K_{\omega}\right)$. So let $G_{\omega}, G_{\omega+1}, \ldots, G_{\tau}$ be the spanning subgraphs of $H=G \backslash K_{\omega}$ corresponding to the vertices $v_{\omega}, v_{\omega+1}, \ldots, v_{\tau}$ of $C$, having vertex set same as $H$ and edge sets defined as follows.

$$
\begin{aligned}
& E\left(G_{\omega}\right)=\left\{v_{\omega} v_{t}: v_{t} \in N\left(v_{\omega}\right) \backslash\left\{v_{1}, v_{2}, \ldots, v_{\omega-1}\right\}\right\} \\
& E\left(G_{\omega+1}\right)=\left\{v_{\omega+1} v_{t}: v_{t} \in N\left(v_{\omega+1}\right) \backslash\left\{v_{1}, v_{2}, \ldots, v_{\omega}\right\}\right\}
\end{aligned}
$$

and in general

$$
E\left(G_{i}\right)=\left\{v_{i} v_{t}: v_{t} \in N\left(v_{i}\right) \backslash\left\{v_{1}, v_{2}, \ldots, v_{i-1}\right\}\right\}, \quad i=\omega, \omega+1, \ldots, \tau .
$$

For $i \in\{\omega, \omega+1, \ldots, \tau\}$, let $m_{i}=\left|E\left(G_{i}\right)\right|$. Clearly $E(H)=E\left(G_{\omega}\right) \cup E\left(G_{\omega+1}\right) \cup \cdots \cup E\left(G_{\tau}\right)$ and $G_{i}=K_{1, m_{i}} \cup\left(n(H)-m_{i}-1\right) K_{1}$, for all $i \in\{\omega, \omega+1, \ldots, \tau\}$. Also, it is clear that

$$
Q(H)=Q\left(G_{\omega}\right)+Q\left(G_{\omega+1}\right)+\cdots+Q\left(G_{\tau}\right) .
$$

The signless Laplacian spectrum of $G_{i}=K_{1, m_{i}} \cup\left(n(H)-m_{i}-1\right) K_{1}$ is

$$
\left\{m_{i}+1,1^{\left[n\left(G_{i}\right)-2\right]}, 0^{\left[n(H)-m_{i}\right]}\right\} .
$$

Therefore,

$$
S_{k}^{+}\left(G_{i}\right)=m_{i}+k, \text { for all } i=\omega, \omega+1, \ldots, \tau .
$$

Now, applying Lemma 1 to Equation (4) and using Equation (5) and the fact that $\sum_{j=\omega}^{\tau} m_{j}=$ $m(H)=m-\frac{\omega(\omega-1)}{2}$, we have

$$
\begin{aligned}
S_{k}^{+}(H) & =\sum_{i=1}^{k} q_{i}(H) \leq \sum_{j=\omega}^{\tau} \sum_{i=1}^{k} q_{i}\left(G_{j}\right)=\sum_{j=\omega}^{\tau} S_{k}^{+}\left(G_{j}\right) \\
& =\sum_{j=\omega}^{\tau}\left(m_{j}+k\right)=m-\frac{\omega(\omega-1)}{2}+(\tau-\omega+1) k .
\end{aligned}
$$


This shows that

$$
S_{k}^{+}\left(G \backslash K_{\omega}\right)=S_{k}^{+}(H) \leq m-\frac{\omega(\omega-1)}{2}+(\tau-\omega+1) k
$$

Therefore, it follows that

$$
\begin{aligned}
S_{k}^{+}(G) & \leq \omega(k+1)-2 k+S_{k}^{+}\left(G \backslash K_{\omega}\right) \\
& \leq \omega(k+1)-2 k+m-\frac{\omega(\omega-1)}{2}+(\tau-\omega+1) k \\
& =k(\tau-1)+m-\frac{\omega(\omega-3)}{2} .
\end{aligned}
$$

Equality occurs in (3) if and only if all the inequalities above become equalities. Since $G$ is connected equality occurs in $S_{k}^{+}(G) \leq S_{k}^{+}\left(K_{\omega}\right)+S_{k}^{+}\left(G \backslash K_{\omega}\right)$, only if $G \cong K_{n}$. Conversely, if $G \cong K_{n}$, then $\tau=n-1, \omega=n, m=\frac{n(n-1)}{2}$ and so equality holds in (3), completing the proof.

Remark 1. For a graph $G \in \Gamma_{1}$, it is easy to see that the upper bound given by (3) is better than the upper bound given by (1) for all $m \geq k(\tau-\omega+1)+\frac{\omega(\omega-1)}{2}$. In particular, for the graph with $\tau=\omega$ and $k \leq n-\omega$, the upper bound (3) is better than the upper bound (1).

Remark 2. Clearly for the graph $G \in \Gamma_{1}$ the upper bound given by (3) is always better than the upper bound given by (2).

Let $\Gamma_{2}$ be the family of all connected graphs except for the graphs $G$, where the vertices in the vertex covering set $S=\left\{v_{1}, v_{2}, \ldots, v_{\left\lfloor\frac{d}{2}\right\rfloor}\right\}$ of the subgraph $P_{d}$ has the property that there are pendent vertices incident at some $v_{i} \in S$ or any two vertices of $S$ forms a triangle with a vertex $v \in V(G) \backslash C$, where $C$ is the vertex covering set of $G$.

Rocha et al. [15] obtained an upper bound for $S_{k}(G)$ in terms of diameter of the graph $G$. Using similar analysis, the following upper bound can be obtained for $S_{k}^{+}(G)$, in terms of the diameter $d-1$ of the graph $G$.

$$
S_{k}^{+}(G) \leq 2(m-d)+1-n+4 k+p+\cos \left(\frac{k \pi}{d}\right)+\frac{\cos \left(\frac{\pi}{d}\right) \sin \left(\frac{k \pi}{d}\right)+\sin \left(\frac{k \pi}{d}\right)}{\sin \left(\frac{\pi}{d}\right)},
$$

where $p$ is the number of isolated vertices in the graph obtained by removing the edges of $P_{d}$ from $G$.

The following theorem gives an upper bound for $S_{k}^{+}(G)$, in terms of the diameter, the number of edges $m$ and the vertex covering number $\tau$ of the graph $G$.

Theorem 3. Let $G \in \Gamma_{2}$ be a connected graph of order $n \geq 3$ with $m$ edges having diameter $d-1$ and vertex covering number $\tau$. Then for $1 \leq k \leq n$,

$$
S_{k}^{+}(G) \leq\left(\tau-\left\lfloor\frac{d}{2}\right\rfloor+2\right) k+m-d+\cos \left(\frac{k \pi}{d}\right)+\frac{\cos \left(\frac{\pi}{d}\right) \sin \left(\frac{k \pi}{d}\right)+\sin \left(\frac{k \pi}{d}\right)}{\sin \left(\frac{\pi}{d}\right)},
$$

with equality if and only if $G \cong P_{n}$. 
Proof. Let $G$ be a connected graph with diameter $d-1$ and vertex cover number $\tau$ and let $C=\left\{v_{1}, v_{2}, \ldots, v_{\tau}\right\}$ be a minimum vertex covering set in $G$. Since the diameter of $G$ is $d-1$, it follows that $P_{d}$ is a subgraph of G. Also, the vertex covering number of a path graph $P_{n}$ on $n$ vertices is $\left\lfloor\frac{n}{2}\right\rfloor$. Let $v_{1}, v_{2}, \ldots, v_{\left\lfloor\frac{d}{2}\right\rfloor}$ be the vertices in $C$, which belong to $V\left(P_{d}\right)$. The signless Laplacian spectrum of $P_{d}$ is $\left\{2-2 \cos \left(\frac{\pi j}{d}\right), 0: j \in\{1,2, \ldots, d-1\}\right\}$. If we remove the edges of $P_{d}$ from $G$, the signless Laplacian matrix of $G$ can be decomposed as

$$
Q(G)=Q\left(P_{d} \cup(n-d-1) K_{1}\right)+Q\left(G \backslash P_{d}\right),
$$

where $G \backslash P_{d}$ is the graph obtained from $G$ by removing the edges of $P_{d}$. Applying Lemma 1 and using the fact that $S_{k}^{+}\left(P_{d} \cup(n-d-1) K_{1}\right)=S_{k}^{+}\left(P_{d}\right)$, we have

$$
\begin{aligned}
S_{k}^{+}(G) & =\sum_{i=1}^{k} q_{i}(G) \leq \sum_{i=1}^{k} q_{i}\left(P_{d}\right)+\sum_{i=1}^{k} q_{i}\left(G \backslash P_{d}\right)=S_{k}^{+}\left(P_{d}\right)+S_{k}^{+}\left(G \backslash P_{d}\right) \\
& =\sum_{j=0}^{k-1}\left(2-2 \cos \left(\frac{\pi(d-j-1)}{d}\right)\right)+S_{k}^{+}\left(G \backslash P_{d}\right) \\
& =2 k+\cos \left(\frac{k \pi}{d}\right)+\frac{\cos \left(\frac{\pi}{d}\right) \sin \left(\frac{k \pi}{d}\right)+\sin \left(\frac{k \pi}{d}\right)}{\sin \left(\frac{\pi}{d}\right)}-1+S_{k}^{+}\left(G \backslash P_{d}\right),
\end{aligned}
$$

where we have used the well-known equality

$$
\sum_{j=0}^{k-1} \cos (n j)=\frac{\sin (n k) \cos (n)+\sin (n k)}{2 \sin (n)}-\frac{1}{2} \cos (n k)+\frac{1}{2} .
$$

In order to establish the result, we need to estimate $S_{k}^{+}\left(G \backslash P_{d}\right)$.

Let $G_{\left\lfloor\frac{d}{2}\right\rfloor+1}, G_{\left\lfloor\frac{d}{2}\right\rfloor+2}, \ldots, G_{\tau}$ be the spanning subgraphs of $H=G \backslash P_{d}$ corresponding to the vertices $v_{\left\lfloor\frac{d}{2}\right\rfloor+1}, v_{\left\lfloor\frac{d}{2}\right\rfloor+2}, \ldots, v_{\tau}$ of $C$, having vertex set same as $H$ and edge sets defined as follows.

$$
E\left(G_{i}\right)=\left\{v_{i} v_{t}: v_{t} \in N\left(v_{i}\right) \backslash\left\{v_{1}, v_{2}, \ldots, v_{i-1}\right\}\right\}, \quad i=\left\lfloor\frac{d}{2}\right\rfloor+1,\left\lfloor\frac{d}{2}\right\rfloor+2, \ldots, \tau .
$$

Now, proceeding similarly as in Theorem 2 , we obtain

$$
S_{k}^{+}\left(G \backslash P_{d}\right) \leq k\left(\tau-\left\lfloor\frac{d}{2}\right\rfloor\right)+m-d+1 .
$$

Therefore, from above we have

$$
\begin{aligned}
S_{k}^{+}(G) & \leq 2 k+\cos \left(\frac{k \pi}{d}\right)+\frac{\cos \left(\frac{\pi}{d}\right) \sin \left(\frac{k \pi}{d}\right)+\sin \left(\frac{k \pi}{d}\right)}{\sin \left(\frac{\pi}{d}\right)}-1+S_{k}^{+}\left(G \backslash P_{d}\right) \\
& \leq\left(\tau-\left\lfloor\frac{d}{2}\right\rfloor+2\right) k+m-d+\cos \left(\frac{k \pi}{d}\right)+\frac{\cos \left(\frac{\pi}{d}\right) \sin \left(\frac{k \pi}{d}\right)+\sin \left(\frac{k \pi}{d}\right)}{\sin \left(\frac{\pi}{d}\right)},
\end{aligned}
$$

and hence the result follows.

Equality occurs in (7) if and only if all the inequalities above occur as equalities. Since $G$ is connected, the equality in the inequality $S_{k}^{+}(G) \leq S_{k}^{+}\left(P_{d}\right)+S_{k}^{+}\left(G \backslash P_{d}\right)$ can only occur if and only if $G \cong P_{n}$. Conversely, if $G \cong P_{n}$, then $\tau=\left\lfloor\frac{n}{2}\right\rfloor, m=n-1, d=n-1$ and so it can be seen that equality holds in (7), completing the proof. 
Remark 3. For the connected graphs $G \in \Gamma_{2}$, it is easy to see that the upper bound given by (7) is better than the upper bound given by (6) for all $k \leq \frac{m-n-d+1+p}{\tau-\left\lfloor\frac{d}{2}\right\rfloor-2}$. In particular, if $G \in \Gamma_{2}$ is such that $\tau \leq\left\lfloor\frac{d}{2}\right\rfloor+2$ and $m \geq n+d-1-p$, the upper bound (7) is always better than the upper bound (6).

Let $\Gamma_{3}$ be the family of all connected graphs except for the graphs $G$, where the vertices in the vertex set $S=\left\{v_{1}, v_{2}, \ldots, v_{s_{1}}, u_{1}, u_{2}, \ldots u_{s_{2}}\right\}$ of the subgraph $K_{s_{1}, s_{2}}, s_{1} \leq s_{2}$, has the property that there are pendent vertices incident at some $v_{i}$ or $u_{j} \in S$ or any two vertices of $S$ forms a triangle with a vertex $v \in V(G) \backslash C$, where $C$ is the vertex covering set of $G$.

Let $K_{s_{1}, s_{2}} s_{1} \leq s_{2}$, be the maximal complete bipartite subgraph of a graph $G$. Using the fact that the vertex covering number of $K_{s_{1}, s_{2}} s_{1} \leq s_{2}$, is $s_{1}$ and its signless Laplacian spectrum is $\left\{s_{1}+s_{2}, s_{1}^{\left[s_{2}-1\right]}, s_{2}^{\left[s_{1}-1\right]}, 0\right\}$, and proceeding similarly as in Theorem 2, we obtain the following upper bound for $S_{k}^{+}(G)$.

Theorem 4. Let $G \in \Gamma_{3}$ be a connected graph of order $n \geq 2$ with $m$ edges having vertex covering number $\tau$. If $K_{s_{1}, s_{2}} s_{1} \leq s_{2}$, is the maximal complete bipartite subgraph of the graph $G$, then

$$
S_{k}^{+}(G) \leq k\left(\tau+s_{2}-s_{1}\right)+m-s_{1}\left(s_{2}-1\right),
$$

with equality if and only if $G \cong K_{s_{1}, s_{2}}$ and $s_{1}+s_{2}=n$.

If $s_{1}=s_{2}$, for the graphs $G \in \Gamma_{3}$, it is easy to see that the upper bound (8) is always better than the upper bound (2).

\section{CONJECTURE 1 IS TRUE FOR SOME MORE CLASSES OF GRAPHS}

In this section, we show that Conjecture 1 holds for some more classes of graphs.

Theorem 5. If $G \in \Gamma_{1}$ is a connected graph of order $n \geq 12$ with $m$ edges having clique number $\omega$, then for $\omega \geq \frac{3+\sqrt{3 n^{2}-14 n+9}}{2}$,

$$
S_{k}^{+}(G) \leq m+\frac{k(k+1)}{2}
$$

for all $k \in\left\{1,2, \ldots,\left\lfloor\frac{n}{2}\right\rfloor\right\}$.

Proof. Let $G$ be a connected graph of order $n$ having clique number $\omega$ and vertex covering number $\tau$. If $\tau=n-1$, clearly $G \cong K_{n}$ and so Conjecture 1 always holds (this is due to the fact that Conjecture 1 holds for all regular graphs). So suppose that $\tau \leq n-2$. With this choice of $\tau$, from inequality (3), we have

$$
S_{k}^{+}(G) \leq k(n-3)+m-\frac{\omega(\omega-3)}{2} \leq m+\frac{k(k+1)}{2},
$$

if $k(2 n-6) \leq k^{2}+k+\omega(\omega-3)$. That is, $k^{2}-(2 n-7) k+\omega(\omega-3) \geq 0$.

Consider the polynomial $f(k)=k^{2}-(2 n-7) k+\omega(\omega-3), k \in[1, n-1]$. The roots of this polynomial are

$$
\alpha=\frac{(2 n-7)+\sqrt{4 n^{2}-28 n+49-4 \omega(\omega-3)}}{2}
$$


and

$$
\beta=\frac{(2 n-7)-\sqrt{4 n^{2}-28 n+49-4 \omega(\omega-3)}}{2} .
$$

Thus $f(k) \geq 0$, for all $k \in(-\infty, \beta] \cup[\alpha,+\infty)$. We will show $\beta \geq \frac{n}{2}$. We have $\beta \geq \frac{n}{2}$ implies

$$
\frac{(2 n-7)-\sqrt{4 n^{2}-28 n+49-4 \omega(\omega-3)}}{2} \geq \frac{n}{2}
$$

which implies that $(n-7)^{2} \geq 4 n^{2}-28 n+49-4 \omega(\omega-3)$, and further implies that $4 \omega^{2}-$ $12 \omega-\left(3 n^{2}-14 n\right) \geq 0$, which gives $\omega \geq \frac{3+\sqrt{3 n^{2}-14 n+9}}{2}$.

Since $\alpha\left(\frac{3+\sqrt{3 n^{2}-14 n+9}}{2}\right)=\frac{3 n-14}{2} \geq n-1$, for all $n \geq 12$, it follows that $\alpha(\omega) \geq n-1$, for all $\omega \leq \frac{3+\sqrt{3 n^{2}-14 n+9}}{2}$. Thus, if $\omega \geq \frac{3+\sqrt{3 n^{2}-14 n+9}}{2}$, we have proved that Conjecture 1 holds for all $k \in\left\{1,2, \ldots,\left\lfloor\frac{n}{2}\right\rfloor\right\}$.

Let $\Omega_{n}$ be a family of those connected graphs $G \in \Gamma_{1}$ for which the vertex covering number $\tau \in\{\omega-1, \omega, \omega+1\}$, that is,

$$
\Omega_{n}=\left\{G \in \Gamma_{1}: \tau=\omega-1 \text { or } \omega \text { or } \omega+1\right\} .
$$

For the family of graphs $\Omega_{n}$, we have the following observation.

Theorem 6. If $G \in \Omega_{n}$, then

$$
S_{k}(G) \leq m+\frac{k(k+1)}{2}
$$

holds for all $k$, if $\tau=\omega-1$; holds for all $k$ except for $k=\omega-2, \omega-1$ if $\tau=\omega$; holds for all $k, k \leq \frac{2 \omega-1-\sqrt{8 \omega+1}}{2}$ and $k \geq \frac{2 \omega-1+\sqrt{8 \omega+1}}{2}$, if $\tau=\omega+1$.

Proof. Let $G \in \Omega_{n}$. Then $\tau \in\{\omega-1, \omega, \omega+1\}$. If $\tau=\omega-1$, from inequality (3), we have

$$
S_{k}^{+}(G) \leq k(\omega-2)+m-\frac{\omega(\omega-3)}{2} \leq m+\frac{k(k+1)}{2},
$$

if $2 k(\omega-2) \leq k^{2}+k+\omega^{2}-3 \omega$, that is,

$$
k^{2}-(2 \omega-5) k+\omega^{2}-3 \omega \geq 0 .
$$

For the polynomial $f(k)=k^{2}-(2 \omega-5) k+\omega^{2}-3 \omega$, the discriminant $D=(2 \omega-5)^{2}-$ $4\left(\omega^{2}-3 \omega\right)=25-8 \omega<0$, if $\omega \geq 4$. This shows that (9) holds for all $\omega \geq 4$. By direct calculations, it can be seen that (9) holds for $\omega \leq 3$. Thus, it follows that (9) is true for all $k$.

If $\tau=\omega$, from inequality (3), we have

$$
S_{k}^{+}(G) \leq k(\omega-1)+m-\frac{\omega(\omega-3)}{2} \leq m+\frac{k(k+1)}{2}
$$

if $2 k(\omega-1) \leq k^{2}+k+\omega^{2}-3 \omega$, that is,

$$
k^{2}-(2 \omega-3) k+\omega^{2}-3 \omega \geq 0 .
$$

For the polynomial $f(k)=k^{2}-(2 \omega-3) k+\omega^{2}-3 \omega$, the roots are $\omega-3$ and $\omega$. It follows that $f(k)<0$, for all $k \in(\omega-3, \omega)$. Since $k$ and $\omega$ are integers and the only integers in $(\omega-3, \omega)$ are $\omega-2, \omega-1$, it follows that $f(k) \geq 0$ for all $k$ except $k=\omega-2, \omega-1$. Thus, it follows that (10) holds for all $k \notin\{\omega-2, \omega-1\}$.

If $\tau=\omega+1$, proceeding similarly as above, it can be seen that the conjecture holds for all $k, k \leq \frac{2 \omega-1-\sqrt{8 \omega+1}}{2}$ and $k \geq \frac{2 \omega-1+\sqrt{8 \omega+1}}{2}$. 
Theorem 7. Let $G \in \Gamma_{2}$ be a connected graph of order $n \geq 2$ with $m$ edges having vertex covering number $\tau$. Let $K_{s_{1}, s_{1}}$ be the maximal complete bipartite subgraph of $G$. Then Conjecture 1 holds for all $k$, if $\tau \leq \frac{1+\sqrt{8 s_{1}\left(s_{1}-1\right)}}{2}$ holds for all $k \leq \frac{2 \tau-1-\sqrt{(2 \tau-1)^{2}-8 s_{1}\left(s_{1}-1\right)}}{2}$ and $k \geq \frac{2 \tau-1+\sqrt{(2 \tau-1)^{2}-8 s_{1}\left(s_{1}-1\right)}}{2}$, if $\tau \geq \frac{1+\sqrt{8 s_{1}\left(s_{1}-1\right)}}{2}$.

Proof. Using $s_{1}=s_{2}$ in inequality (8), we have

$$
S_{k}^{+}(G) \leq k \tau+m-s_{1}\left(s_{1}-1\right) \leq m+\frac{k(k+1)}{2},
$$

if

$$
k^{2}-(2 \tau-1) k+2 s_{1}\left(s_{1}-1\right) \geq 0 .
$$

The roots of the polynomial $f(k)=k^{2}-(2 \tau-1) k+2 s_{1}\left(s_{1}-1\right)$ are $\alpha=\frac{2 \tau-1+\sqrt{\theta}}{2}$ and $\beta=$ $\frac{2 \tau-1-\sqrt{\theta}}{2}$, where $\theta=(2 \tau-1)^{2}-8 s_{1}\left(s_{1}-1\right)$. We have $(2 \tau-1)^{2}-8 s_{1}\left(s_{1}-1\right) \leq 0$, which implies that $4 \tau^{2}-4 \tau-\left(8 s_{1}^{2}-8 s_{1}-1\right) \leq 0$, which gives $\tau \leq \frac{1+\sqrt{8 s_{1}\left(s_{1}-1\right)}}{2}$. This shows that the discriminant of the polynomial $f(k)$ is non-positive for all $\tau \leq \frac{1+\sqrt{8 s_{1}\left(s_{1}-1\right)}}{2}$. That is, (11) holds for all $\tau \leq \frac{1+\sqrt{8 s_{1}\left(s_{1}-1\right)}}{2}$. On the other hand if the discriminant of the polynomial $f(k)$ is non-negative, then (11) holds for all $k \geq \alpha$ and for all $k \leq \beta$, completing the proof.

Let $G$ be a connected bipartite graph of order $n$ having the vertex covering number $\tau$. For bipartite graphs, it is well known that $\tau \leq \frac{n}{2}$. With this in mind, we have the following observation for bipartite graphs.

Theorem 8. Let $G \in \Gamma_{3}$ be a connected bipartite graph of order $n \geq 4$ with $m$ edges having the vertex covering number $\tau$. If $K_{s_{1}, s_{1}}$, with $s_{1} \geq \frac{n}{4}$, is the maximal complete bipartite subgraph of the graph $G$, then

$$
S_{k}(G) \leq m+\frac{k(k+1)}{2}
$$

for all $k \leq \frac{n}{7}-1$ and $k \geq \frac{6 n}{7}$.

Proof. Using $s_{1}=s_{2}$ in (8) and the fact that $\tau \leq \frac{n}{2}$, for bipartite graphs we have

$$
S_{k}^{+}(G) \leq k \tau+m-s_{1}\left(s_{1}-1\right) \leq k\left(\frac{n}{2}\right)+m-s_{1}\left(s_{1}-1\right) \leq m+\frac{k(k+1)}{2}
$$

if

$$
k n \leq k(k+1)+2 s_{1}\left(s_{1}-1\right) .
$$

The right hand side of (10) is an increasing function of $s_{1}$. Therefore, to prove the assertion, it suffices to consider $s_{1}=\frac{n}{4}$. With this value of $s_{1}$, from (12), we have

$$
k^{2}-(n-1) k+\frac{n(n-4)}{8} \geq 0 \text {. }
$$

The roots of the polynomial $f(k)=k^{2}-(n-1) k+\frac{n(n-4)}{8}$ are

$$
\alpha=\frac{n-1+\sqrt{0.5 n^{2}+1}}{2}, \quad \beta=\frac{n-1-\sqrt{0.5 n^{2}+1}}{2} .
$$

This shows that $f(k) \geq 0$, for all $k \geq \alpha$; and $f(k) \geq 0$, for all $k \leq \beta$. By using elementary algebra it can be seen that $\alpha<0.8535 n$ and $\beta>0.1464 n-1$. Hence the result follows. 
For graphs with girth $g \geq 5$, Rocha et al. [15] showed that Brouwer's conjecture holds for all $k \leq\left\lfloor\frac{g}{5}\right\rfloor$. Using similar analysis, we have the following observation.

Theorem 9. For connected graphs with girth $g \geq 5$, Conjecture 1 holds for all $k, 1 \leq k \leq\left\lfloor\frac{g}{5}\right\rfloor$.

Using Theorem 3, the fact that

$$
\cos \left(\frac{k \pi}{d}\right)+\frac{\cos \left(\frac{\pi}{d}\right) \sin \left(\frac{k \pi}{d}\right)+\sin \left(\frac{k \pi}{d}\right)}{\sin \left(\frac{\pi}{d}\right)} \leq 2 k+1
$$

and proceeding similarly as in above theorems, we arrive at the following observation.

Theorem 10. Let $G \in \Gamma_{2}$ be a connected graph of order $n \geq 3$ with $m$ edges having diameter $d-1$ and vertex covering number $\tau$. Then for $1 \leq k \leq n$, Conjecture 1 holds for all $k$, if $\tau \leq \frac{2\left\lfloor\frac{d}{2}\right\rfloor-7+\sqrt{8(d-1)}}{2}$; holds for all $k$,

$$
k \leq \frac{2 \tau-2\left\lfloor\frac{d}{2}\right\rfloor+7-\sqrt{2 \tau-2\left\lfloor\frac{d}{2}\right\rfloor+7-8(d-1)}}{2}
$$

and

$$
k \geq \frac{2 \tau-2\left\lfloor\frac{d}{2}\right\rfloor+7+\sqrt{2 \tau-2\left\lfloor\frac{d}{2}\right\rfloor+7-8(d-1)}}{2},
$$

if $\tau \geq \frac{2\left\lfloor\frac{d}{2}\right\rfloor-7+\sqrt{8(d-1)}}{2}$.

\section{CONCLUDING REMARKS}

The aim of this paper is twofold. Firstly, in Section 2, we obtained some upper bounds for the graph invariant $S_{k}^{+}(G)$, in terms of clique number $\omega$, the vertex covering number $\tau$ and the diameter of the graph $G$. These bounds can be used to obtain the upper bounds for the signless Laplacian energy of the graph $G$ and so can be helpful to obtain the extremal graphs among various families of the graphs. Secondly, in Section 3, we have used the results of Section 2 to verify the truth of the Conjecture 1 for some more families of graphs. Although, in Sections 2 and 3, we have restricted ourselves to graphs $G \in\left\{\Gamma_{1}, \Gamma_{2}, \Gamma_{3}\right\}$, the importance of these results can be realized from the fact that not many families of graphs are known for which Conjecture 1 holds.

Acknowledgements. The authors are grateful to the anonymous referee for his valuable comments and suggestions. The research of S. Pirzada (first author) is supported by SERBDST, New Delhi under the research project number MTR/2017/000084.

\section{REFERENCES}

[1] Ashraf F., Omidi G.R., Tayfeh-Rezaie B. On the sum of signless Laplacian eigenvalues of a graph. Linear Algebra Appl. 2013, 438, 4539-4546. doi:10.1016/j.laa.2013.01.023

[2] Bai H. The Grone-Merris conjecture. Trans. Amer. Math. Soc. 2011, 363 4463-4474. doi:10.1.1.360.9876

[3] Brouwer A.E., Haemers W.H. Spectra of graphs. http://homepages.cwi.nl/aeb/math/ipm.pdf

[4] Cvetkovic D., Doob M., Sachs H. Spectra of Graphs-Theory and Application. Academic Press, New York, 1980. 
[5] Das K.C., Mojallal S.A., Gutman I. On Laplacian energy in terms of graph invariants. Applied Mathematics and Computation 2015, 268, 83-92. doi:10.1016/j.amc.2015.06.064

[6] Fulton W. Eigenvalues, invariant factors, highest weights and Schubert calculus. Bull. Amer. Math. Soc. (NS) 2000, 37, 209-249. doi:10.1090/S0273-0979-00-00865-X

[7] Ganie H.A., Pirzada S. On the bounds for signless Laplacian energy of a graph. Discrete Appl. Math. 2017, 228, 3-13. doi:10.1016/j.dam.2016.09.030

[8] Ganie H.A., Alghamdi A.M., Pirzada S. On the sum of the Laplacian eigenvalues of a graph and Brouwer's conjecture, Linear Algebra Appl. 2016, 501, 376-389. doi:10.1016/j.laa.2016.03.034

[9] Ganie H.A., Pirzada S. Corrigendum to "On the sum of the Laplacian eigenvalues of a graph and Brouwer's conjecture". Linear Algebra Appl. 2018, 538, 228-230. doi:10.1016/j.laa.2017.10.020

[10] Ganie H.A., Pirzada S., Rezwan Ul Shaban, Li X. Upper bounds for the sum of Laplacian eigenvalues of a graph and Brower's conjecture. Discrete Math. Algorithms Appl. 2019, 11 (2), (15 pages). doi:10.1142/S1793830919500289

[11] Ganie H.A., Pirzada S., Rather B.A., Trevisan V. Further developments on Brouwer's conjecture for the sum of Laplacian eigenvalues of graphs. Linear Algebra Appl. 2020, 588, 1-18. doi:10.1016/j.laa.2019.11.020

[12] Grone R., Merris R. The Laplacian spectrum of a graph II. SIAM J. Discrete Math. 1994, 7, $221-229$.

[13] Pirzada S. An Introduction to Graph Theory. Universities Press, OrientBlackSwan, Hyderabad, 2012.

[14] Pirzada S., Ganie H. A. On the Laplacian eigenvalues of a graph and Laplacian energy. Linear Algebra Appl. 2015, 486, 454-468. doi:10.1016/j.laa.2015.08.032

[15] Rocha I., Trevisan V. Bounding the sum of the largest Laplacian eigenvalues of graphs. Discrete Appl. Math. 2014, 170, 95-103. doi:10.1016/j.dam.2014.01.023

[16] Yang J., You L. On a conjecture for the signless Laplacian eigenvalues. Linear Algebra Appl. 2014, 446, 115-132. doi:10.1016/j.laa.2013.12.032

Received 14.10.2018

Пірзада С., Гані Х.А., Альгамді А.М. Про суму беззнакових лапласіанівських спектрів графів // Карпатські матем. публ. - 2019. - Т.11, №2. — С. 407-417.

$\Delta$ ля деякого простого графа $G(V, E)$ з $n$ вершинами і $m$ ребрами, множиною вершин $V(G)=\left\{v_{1}, v_{2}, \ldots, v_{n}\right\}$ і множиною ребер $E(G)=\left\{e_{1}, e_{2}, \ldots, e_{m}\right\}$, матриця суміжності $A=\left(a_{i j}\right)$ графа $G$ - це $(0,1)$-квадратна матриця порядку $n$, для якої елементи з індексом $(i, j)$ дорівнюють 1 , якщо $v_{i}$ суміжна з $v_{j}$ і 0 у протилежному випадку. Нехай $D(G)=\operatorname{diag}\left(d_{1}, d_{2}, \ldots, d_{n}\right)$ - діагональна матриця, асоційована з $G$, ле $d_{i}=\operatorname{deg}\left(v_{i}\right)$, для всіх $i \in\{1,2, \ldots, n\}$. Матриці $L(G)=D(G)-A(G)$ і $Q(G)=D(G)+A(G)$ називаються лапласіанівські і беззнакові лапласіанівські матриці, відповідно, а їх спектри (власні значення), відповідно — лапласіанівським спектром (L-спектром) та беззнаковим лапласіанівським спектром ( $Q$-спектром) графа G. Якшо $0=\mu_{n} \leq \mu_{n-1} \leq \cdots \leq \mu_{1} \in$ лапласіанівські власні значення $G$, Броувер припустив, що сума $k$ найбільших лапласіанівських значень $S_{k}(G)$ задовольняє $S_{k}(G)=\sum_{i=1}^{k} \mu_{i} \leq m+\left(\begin{array}{c}k+1 \\ 2\end{array}\right)$ і це припущення $є$ все ще відкритим. Якщо $q_{1}, q_{2}, \ldots, q_{n}$ - беззнакові лапласіанівські власні значення

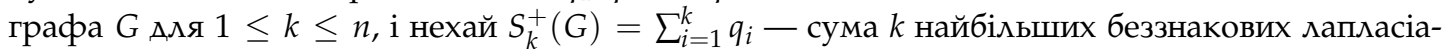
нівських власних значень G. Аналогічно до припущення Броувера, Асхраф та ін. припустили, що $S_{k}^{+}(G) \leq m+\left(\begin{array}{c}k+1 \\ 2\end{array}\right)$ для всіх $1 \leq k \leq n$. Це припущення було підтверджено для деяких класів графів. Ми отримали верхнє обмеження для $S_{k}^{+}(G)$ в термінах клікових чисел $\omega$, чисел покриття вершин $\tau$ і діаметра графа $G$. Зрештою, ми показали, шо припушення виконується Аля широкої сім'ї графрів.

Ключові слова і фрази: беззнакові лапласіанівські спектри, припушення Броувера, клікові числа, числа покриття вершин, діаметр. 